\title{
ASYMPTOTIC ENUMERATION OF LATIN RECTANGLES
}

\author{
BY C. D. GODSIL AND B. D. MCKAY
}

A $k \times n$ Latin rectangle is a $k \times n$ matrix with entries from $\{1,2, \ldots, n\}$ such that no entry occurs more than once in any row or column. (Thus each row is a permutation of the integers $1,2, \ldots, n$.) Let $L(k, n)$ be the number of $k \times n$ Latin rectangles. An outstanding problem is to determine the asymptotic value of $L(k, n)$ as $n \rightarrow \infty$, with $k$ bounded by a suitable function of $n$.

The first attack on this problem was made by Erdös and Kaplansky [1], who obtained the correct value for $k=O\left((\log n)^{3 / 2-\epsilon}\right)$. The range of validity was later widened to $k=o\left(n^{1 / 3}\right)$ by Yamamoto [8] and to $k=o\left(n^{1 / 2}\right)$ by Stein [7]. We have obtained the correct value for $k=o\left(n^{6 / 7}\right)$, and at the same time have sharpened the known approximations for fixed $k \geq 4$. Specifically, we have the following Theorem.

TheOREM. Let $k=O\left(n^{1-\delta}\right)$ for some fixed $\delta>0$. Then

$$
L(k, n)=\frac{(n !)^{n+k}}{n^{n k}(n-k) ! n} \exp (k(k-1) l(k, n)),
$$

where

$$
\begin{aligned}
l(k, n) & =\frac{1}{4 n}+\frac{k-1}{6 n^{2}}+\frac{k^{2}-k-1}{8 n^{3}}+\frac{12 k^{3}-13 k^{2}-13 k-6}{120 n^{4}} \\
& +\frac{15 k^{4}-18 k^{3}-18 k^{2}-28 k+47}{180 n^{5}}+O\left(\frac{k^{5}}{n^{6}}\right) .
\end{aligned}
$$

The bound $l(k, n) \geq 0$ is a consequence of the van der Waerden permanent conjecture. It is interesting to note that the leading coefficients of the expansion of $l(k, n)$ are in harmonic progression. If this trend continues (which we cannot prove) it would suggest that

$$
L(k, n) \sim \frac{(n !)^{n+k}}{n^{n k}(n-k) ! n}\left(1-\frac{k}{n}\right)^{-n / 2} e^{-k / 2}
$$

as $n \rightarrow \infty$ with $k=O\left(n^{1-\delta}\right)$.

As with previous work, our Theorem is obtained by first estimating the average number of ways in which a $k \times n$ rectangle can be extended to a $(k+1) \times n$ rectangle by adding an extra row. The important new feature of our work is that is uses some of the recently developed theory concerning the matchings and rook polynomials [2-5].

Received by the editors March 30, 1983.

1980 Mathematics Subject Classification. Primary 05A15, 05B20.

(C) 1984 American Mathematical Society $0273-0979 / 84 \$ 1.00+\$ .25$ per page 
From [3 or 5] we know that the number of ways of extending a given $k \times n$ rectangle $R$ can be expressed as $\int_{0}^{\infty} e^{-x} r(x) d x$, where $r(x)$ is a polynomial of degree $n$ determined by $R$. Now, all the zeros of $r(x)$ are known [4, Lemma 4.1 and Theorem 4.3] to lie in the real interval [0,4k-4). For $n \gg k$, this implies that the integrand is concentrated in a fairly small region near $x=n$. Moreover, the moments of the set of zeros of $r(x)$ enumerate a certain family of closed walks in a $k$-regular bipartite graph $G$ associated with $R$ [2]. By comparing these with another family of closed walks in $G$ [6], we obtain an accurate estimate of the number of extensions of $R$ in terms of the counts of certain small subgraphs (squares, etc.) in $G$. The average values of these counts are then estimated by another method and the Theorem follows.

A similar technique has been used to asymptotically enumerate disjoint perfect matchings in the complete graph. Details will appear elsewhere.

\section{REFERENCES}

1. P. Erdös and I. Kaplansky, The asymptotic number of Latin rectangles, Amer. J. Math. 68 (1946), 230-236.

2. C. D. Godsil, Matchings and walks in graphs, J. Graph Theory 5 (1981), 285-297.

3. _ Hermite polynomials and a duality relation for the matchings polynomial, Combinatorica 1 (1981), 257-262.

4. O. J. Heilmann and E. H. Lieb, Theory of monomer-dimer systems, Comm. Math. Phys. 25 (1972), 190-232.

5. S. A. Joni and G.-C. Rota, A vector space analog of permutations with restricted position, J. Combinatorial Theory Ser. A 29 (1980), 59-73.

6. B. D. McKay, The expected eigenvalue distribution of a large regular graph, Linear Algebra Appl. 40 (1981), 203-216.

7. C. M. Stein, Asymptotic evaluation of the number of Latin rectangles, J. Combinatorial Theory Ser. A 25 (1978), 38-49.

8. K. Yamamoto, On the asymptotic number of Latin rectangles, Japan. J. Math. 21 (1951), 113-119.

Department of Mathematics, Simon Fraser University, Burnaby, BC, CanADA V5A 1S6

Department of Computer Science, Vanderbilt University, Nashville, TenNESSEE 37235 